\title{
SUBACTIVISMO FEMINISTA \\ Y REPERTORIOS DE ACCIÓN \\ COLECTIVA DIGITALES: PRACTICAS CIBERFEMINISTAS EN TWITTER
}

\section{Feminist sub-activism and digital collective repertoires: Cyberfeminist practices on Twitter}

\section{José-Manuel Sánchez-Duarte y Diana Fernández-Romero}
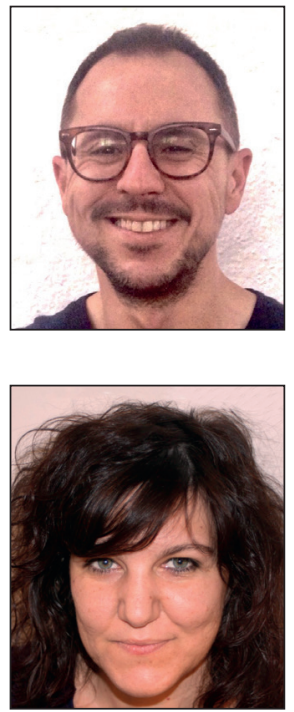

José-Manuel Sánchez-Duarte es doctor en Comunicación por la Universidad Rey Juan Carlos (URJC) de Madrid, y en Ciências Sociais (especialidad Políticas) por la PUC de São Paulo. También realizó el postgrado en Formación de especialistas en investigación social aplicada y análisis de datos por el Centro de Investigaciones Sociológicas. Es profesor de Ciencia política y Gestión pública en la URJC. Su principal línea de investigación se centra en los usos de las tecnologías digitales para la acción política.

http://orcid.org/0000-0002-5627-2713

josemanuel.sanchez@urjc.es

Diana Fernández-Romero es doctora en Ciencias de la Información por la Universidad Complutense de Madrid (UCM). Especialista en Cultura y Violencia de género por la UNED. Docente en la Facultad de Ciencias de la Comunicación de la Universidad Rey Juan Carlos. Su tesis doctoral, "Destrucción y reconstrucción de la identidad de mujeres maltratadas: análisis de discursos autobiográficos y de publicidad institucional" es premio extraordinario de doctorado por la UCM y premio de la Delegación del Gobierno para la Violencia de Género a tesis doctorales sobre violencia de género.

http://orcid.org/0000-0003-2692-4474 diana.fernandez.romero@urjc.es

Universidad Rey Juan Carlos Campus de Fuenlabrada, Edificio Departamental I, Despacho 220 Fuenlabrada (Madrid), España

\section{Resumen}

La Red se conforma como un emergente espacio de militancia política para el activismo feminista. Las prácticas subactivistas, menos institucionalizadas y más conectadas con la experiencia subjetiva, encuentran en la tecnología digital nuevas formas para conectar la política latente y la participación política cívica con el activismo convencional. A partir de 12 entrevistas en profundidad con activistas con amplia trayectoria en el movimiento feminista y un uso intensivo de las redes sociales, en particular Twitter, para sus iniciativas políticas, se intentan definir perfiles y prácticas de uso. Como conclusión se muestra que los repertorios de acción colectiva digitales establecen un continuum entre las militancias offline y online, así como amplifican, potencian y democratizan la divulgación feminista. A su vez, las redes sociales se configuran como espacios endogámicos y no conquistados del todo por los colectivos feministas, por lo que se hace necesario crear novedosos escenarios propios y potenciar propuestas y discursos con capacidad política transformadora.

\section{Palabras clave}

Política, Comunicación política; Activismo; Activismo digital; Subpolítica; Subactivismo; Feminismo; Ciberfeminismo; Twitter.

\section{Abstract}

The Network is an emerging space for political and feminist activism. Sub-activist practices, less institutionalized and more connected with subjective experiences, find in digital technology new ways of connecting latent politics and civic political participation with conventional activism. We try to define profiles and practices of use from 12 in-depth interviews with activists who have a long history in the feminist movement and who use social networks intensively, in particular Twitter, for their policy initiatives. As a conclusion this article shows how digital collective repertoires of action establish a continuum between real life and online militancy as well as amplify, empower, and democratize feminist popularization. In turn, social 
networks are configured as inbred spaces and not fully conquered by feminist groups, so it is necessary to create novel scenarios of their own and to promote proposals and discourses with transformative political capacity.

\section{Keywords}

Politics; Political communication; Activism; Digital activism; Sub-politics; Sub-activism; Feminism; Cyberfeminism; Twitter.

Sánchez-Duarte, José-Manuel; Fernández-Romero, Diana (2017). "Subactivismo feminista y repertorios de acción colectiva digitales: prácticas ciberfeministas en Twitter". El profesional de la información, v. 26, n. 5, pp. 894-902.

https://doi.org/10.3145/epi.2017.sep.11

\section{Introducción}

\subsection{De la subpolítica al subactivismo. Ciudadanía la- tente y organizaciones híbridas}

La definición y la práctica de la política han superado los límites tradicionales. Las organizaciones clásicas de representación han ido progresivamente perdiendo peso en la canalización de las demandas de la ciudadanía mientras que los movimientos sociales han sufrido también su propia crisis de legitimidad. Los límites de la noción de política se han ido abriendo hacia formas más flexibles y capaces de establecer vínculos nuevos, dando lugar a contextos emergentes (Beck, 2002, p. 61). Desde este punto de vista, la subpolítica, entendida como la política que existe al margen y más allá de las instituciones, no tiene una relevancia menor sino un grado de institucionalización menos importante que la política tradicional (Holzer; Sorensen, 2003).

\section{La subpolítica, entendida como la que existe al margen y más allá de las insti- tuciones, no tiene una relevancia menor sino un grado de institucionalización me- nos importante que la política tradicional}

Algunas áreas que antes no eran consideradas como propias de la acción política, ahora se identifican como novedosos campos de actuación. Estas nuevas áreas son difíciles de analizar utilizando los métodos tradicionales para medir la participación política. El subactivismo es un tipo de política que opera en la "experiencia subjetiva" (Bakardjieva, 2009, p. 92) y con fuerte arraigo en la vida social cotidiana (Tarrow, 2012, p. 37).

Este contexto de acción política genera reglas de participación distintas a las tradicionales. Las definiciones clásicas de acción política por parte de la ciudadanía son por un lado la ausencia de participación (apolítica y antipolítica), y por otro, la participación manifiesta (formal o a través del activismo extraparlamentario). Frente a ellas surge otra política que se materializa a través de la atención (compromiso social) y de la acción (compromiso cívico) (Amnå; Ekman, 2014; Ekman; Amnå, 2012).

La ciudadanía que sigue este tipo de acción política ya no precisa de intermediarios, protocolos de actuación o membresías formales. Por el contrario, se implica en causas, defensas y campañas que están más cercanas a un determi- nado estilo de vida que a un programa político de máximos (Bennett, 1998; Coleman, 2005; Giddens, 1991).

Así surgen nuevos tipos de organizaciones, que han sido denominadas híbridas (Chadwick, 2007, p. 288) o postburocráticas (Bimber, 2003). Estas organizaciones están menos institucionalizadas que las convencionales, tienen una pertenencia flexible, presentan pocas barreras de entrada y salida y siguen modos de acción basados en la autoconvocatoria. Como señalan Heany y Rojas (2014, p. 1047), ante las dificultades de movilización, las organizaciones híbridas, que traspasan las fronteras de dos o más movimientos, problemas o identidades, generan fácilmente participación e incluso compromiso.

El funcionamiento de las nuevas organizaciones está muy vinculado al uso de la tecnología, ya que ésta, como indica Benkler (2014, p. 67), hace más viables las prácticas sociales. Se produce así una relación entre las posibilidades de la tecnología y la respuesta de la ciudadanía.

Se considera que las actividades políticas surgen en la superposición de la política y la cultura (Lim; Kann, 2008, p. 92), en este caso digital. Gracias en parte a la cultura digital, el activismo contemporáneo adquiere diferentes roles dependiendo de sus respectivas causas, perfilando una forma de acción modulable, adaptativa y con rasgos tecnológicos propios (Neumayer; Svensson, 2016, p. 144).

\subsection{Repertorios de acción colectiva digital}

El concepto de "repertorio de acción colectiva" se refiere no sólo a aquellas acciones que realiza la ciudadanía cuando interviene en un conflicto con otros, sino también a aquello que sabe hacer y a lo que otros esperan que haga (Tilly, 2009). Según Tarrow (2012, pp. 87), con los cambios políticos, sociales y tecnológicos, los reportorios de acción colectiva han pasado de ser locales, segmentados y particulares, a convertirse en cosmopolitas, modulares y autónomos. La tecnología ha hecho posible la conversión de las formas tradicionales de acción hacia otras acciones en red.

Para identificar los repertorios de acción colectiva en el ámbito digital se precisan tres dimensiones de análisis, que se sintetizan a continuación. La primera fue propuesta por Van-Laer y Van-Aelst (2010) y las dos siguientes por Flanagin, Stohl y Bimber (2006, pp. 34-36):

1. Distinción entre acciones que emplean la Red como soporte para organizar y coordinar acciones "convencionales" como convocatoria de manifestaciones a través de redes sociales, cuestaciones para causas concretas... (internet como 
soporte) y las acciones que ocurren sólo en la Red como hacktivismo... (internet como base).

2. Distinción en el modo de compromiso entre modo emprendedor (en el que la ciudadanía tiene un alto grado de autonomía y puede diseñar una acción sin ser sancionada o controlada por una autoridad central) e institucional (con estructuras predecibles dentro de unos parámetros generales que son más o menos jerárquicos y burocráticos).

3. Distinción en el patrón de interacción, que puede ser personal (con una interacción directa) e impersonal (no directa).

Estos tipos de acción plantean cuestiones de eficacia, potencialidad y límites. En términos optimistas y ante un contexto de desafección política, la ciudadanía emplea la Red como campo de acción para aumentar su compromiso democrático. De esta manera identifica y da a conocer sus fines, solicita y fomenta apoyos, organiza y comunica información o recluta y recauda fondos (Van-Aelst; Walgrave, 2002, p. 488).

La disminución de restricciones a la participación respecto al tiempo, el esfuerzo o el acceso a la información que permite internet, crea incipientes modos de acción más horizontales, autoorganizativos y flexibles, así como comunidades distribuidas, experimentales, amateurs con conciencias compartidas (Treré; Cargnelutti, 2014, p. 185) y lenguajes más abiertos y de fácil reapropiación por parte de los activistas (Baym; Shah, 2011, p. 1.034).

Pero internet también presenta una serie de limitaciones a la acción colectiva. Se pueden señalar las tres siguientes:

1) El elemento numérico o cuantitativo de las acciones. Un número elevado de participantes no significa un aumento del compromiso ni de la calidad de los procesos políticos. La obsesión por reunir gente en torno a acciones provoca cierta obsesión por la convocatoria frente a la reflexión (Mozorov, 2011, p. 195), así como un nivel máximo de participación mínima (Crouch, 2005) en el que la incidencia política puede ser reducida (Robertson; Vatrapu; Medina, 2010, p. 29), las habilidades de los participantes son limitadas (brecha de posicionamiento) (Del- Fresno, 2016) y las narrativas de acción se producen en espacios públicos comercializados (Papacharissi, 2009).

2) Una segunda limitación o crítica es si este tipo de acciones en red contribuyen a la disminución del capital social de las comunidades (Putnam, 1995; Coleman, 1988), erosionando la identidad y solidaridad colectiva y los compromisos a largo plazo. Según esta visión, prevalece un tipo de militante que realiza actos pasivos, inofensivos, personales y de entretenimiento (slacktivism) (Mozorov, 2011), más vinculados con prácticas estéticas, autoexpresivas y performativas que con la capacidad de incidir realmente en el poder y las instituciones.

3) El último de los límites en la acción política en red es el que considera internet como una estructura que favorece lo que se ha denominado "exposición selectiva" (Johnson; Bichard; Zhang, 2009, p. 76): los ciudadanos tienden a reforzar sus ideas preconcebidas sin tener en cuenta los argumentos opuestos, "los del otro lado" (Neuman; Bimber; Hindman, 2010, p. 26). Esta es una pauta que se hereda de los medios de comunicación de masas (Bennett; lyengar,
Esquema 1. Dimensiones de análisis de los repertorios de acción colectiva digital.

1. Internet como soporte

1. Internet como base

2. Modo de compromiso

Emprendedor: alta responsabilidad y oportunidad

3. Modo de interacción

Personal: interacción directa

3. Modo de interacción

Impersonal: interacción no directa

\section{Modo de compromiso}

Institucional: baja responsabilidad y oportunidad

Elaborado a partir de Van-Laer y Van-Aelst (2010) y Flanagin, Stohl y Bimber (2006)

2010 , p. 31), pero que en internet se amplifica. Dicha exposición selectiva da lugar a una esfera pública fragmentada y aislada donde las posiciones de las comunidades se refuerzan más que se critican (Dahlberg, 2007, p. 827), constituyendo "cajas de resonancia" auto-referenciales y con poca disponibilidad para establecer colaboraciones y coaliciones con otros grupos (Mancini, 2013, p. 56). Asimismo la exposición selectiva favorece la polarización (Benkler, 2014, p. 276) y la fragmentación de los discursos (Sunstein, 2007).

La traslación de este contexto a los colectivos y organizaciones de la sociedad civil y movimientos sociales, así como su conversión en movimientos globales, ha dislocado los marcos tradicionales de organización de la protesta: tipos de conflicto, formas de coordinación, modos de producción de identidad compartida y repertorios de acción colectiva (Sádaba, 2012, p. 791). Movimientos pro-derechos civiles, pacifistas o feministas han recurrido a múltiples formas organizativas para canalizar la acción (grupos profesionales a nivel nacional, grupos locales de base...) (McCarthy, 1999, p. 213). Pero, ¿cómo se desarrollan estos colectivos en la Red? ¿Cuáles son sus prácticas y repertorios de acción?

\subsection{Ciberactivismo feminista}

La transformación digital también ha contribuido a que se configuren nuevas formas de activismo político feminista. Como señalan Núñez, Fernández y Peña (2016) diversas autoras como Paterson (1992), Plant (1997), Balka (1996), Wajcman (2000) o Friedman (2003), herederas de Solanas y su SCUM Manifesto (1968) y de Firestone y su The dialect of sex (1968) (2016, p. 179), comprenden que la tecnología es liberadora y supone un impulso para la participación política y social de las mujeres y por tanto para promover su emancipación. Este enfoque utópico del ciberfeminismo entiende que las nuevas tecnologías pueden contribuir a la elaboración de nuevas identidades políticas en la esfera del activismo y a disminuir las desigualdades de género mediante nuevas formas de activismo político e interacción horizontal. Asimismo se entiende que la incorporación de las TIC a las estrategias activistas no sólo ha logrado un mayor alcance, sino que también ha potenciado discursos activistas que han cambiado para su adaptación a la esfera pública digital (Núñez; Fernández; Vázquez, 2015, p. 66). 
El Manifesto for cyborgs de Haraway (1985) afirmaba que la tecnología es una parte esencial de nuestra identidad, que puede ser productivo pensarnos como cyborgs, y utilizar esa conceptualización para cambiar las relaciones entre el poder, la identidad y la tecnología. Braidotti (1994) encuentra la Web como el espacio donde la subjetividad nómada puede conquistar nuevas libertades y crear nuevas comunidades virtuales.

Según Núñez, Vázquez y Fernández (2016, p. 862), antes del cyborg de Haraway, Cockburn (1985) había considerado que la competencia tecnológica es un acto interpretado como la expresión de la identidad masculina y por eso las mujeres muestran resistencia a participar en las tecnologías masculinizadas. Por ello proponía un movimiento autónomo de mujeres que transformara la naturaleza de la tecnología. Más adelante autores como Danitz y Strobel (1999) o Huyer (1999) observan que la participación horizontal o la toma de decisiones descentralizada puede no resultar demasiado ágil y ser contraproducente.

En esta línea Dean (2005) habla de la fetichización de la tecnología, que se articula en torno a una "fantasía" de la participación que fomenta la circulación de mensajes sin contenido político o que se diluyen en el flujo comunicativo de la Red. Para Dean esto contrasta con la idea que tienen los sujetos de que están promoviendo el activismo político por el mero hecho de poner en circulación mensajes con intención activista. La proliferación de discursos informativos que no son contrahegemónicos estaría contribuyendo, según Dean, a la constitución del capitalismo comunicativo. No obstante, en su opinión la comunicación online podría ser efectiva si se realizaran propuestas concretas que necesitan politizarse (Núñez; Fernández; Peña, 2016, pp. 182-183).

En los nuevos espacios de relación online, las comunidades ciberactivistas feministas confeccionan con asiduidad un vínculo estrecho con las comunidades feministas que no están en internet. Estamos hablando por tanto de un proceso de mesomobilización (Scott; Street, 2000) que propicia una alianza estratégica entre redes dentro y fuera de internet para impulsar propuestas activistas.

Diversas obras sobre las comunidades feministas en el contexto español que luchan contra la violencia de género, identifican un continuum desde el activismo que realizan algunas entidades que solamente tienen funcionamiento online, hasta asociaciones que llevan a la práctica su actividad fuera de la Red y utilizan la Web y las redes sociales como escaparate de sus acciones, pasando por aquellas que combinan los fondos virtuales y no virtuales y retroalimentan el activismo y las campañas offline con las online (Núñez; Vázquez; Fernández, 2016, p. 869).

\section{Método}

El objetivo del artículo es comprender si las prácticas activistas feministas online en España pueden entenderse como una forma de subactivismo: un tipo de acciones colectivas basadas en la experiencia subjetiva de las militantes y en el arraigo de esas prácticas en sus rutinas y vidas cotidianas.

Para ello se intenta identificar qué tipo de iniciativas realizan en redes sociales y en concreto en Twitter. La selección de este medio se debe a considerarlo un espacio en el que el discurso activista se articula de forma potente y fluida y donde se utiliza un lenguaje más abierto y de reapropiación más fácil que en otras redes sociales. De igual modo interesa comprender si Twitter está sirviendo de caja de resonancia autorreferencial y endogámica para el activismo feminista en la Red.

El trabajo se basó en la realización de entrevistas en profundidad semiestructuradas. La selección de los sujetos entrevistados se centró en informantes especiales, es decir, personas con información directa y relevante para los propósitos de la investigación y una posición única en la comunidad, grupo u organización a investigar (Vallés, 1999, p. 213). La importancia de seleccionar a estas personas reside en su potencial para ayudar a realizar una comprensión teórica sobre el área estudiada de la vida social (Taylor; Bogdan, 1987, p. 108). En este caso se trataba de activistas con amplia trayectoria de militancia en el movimiento feminista y que realizaban un uso intensivo de las redes sociales, en concreto Twitter, para sus iniciativas políticas.

En total se seleccionaron a 12 activistas a las que se les realizó una entrevista semiestructurada, en algunos casos de manera presencial y en otros a través de Skype. Las 12 mujeres entrevistadas presentaban edades comprendidas entre los 27 y 57 años. Sus ocupaciones laborales eran diversas prevaleciendo los perfiles profesionales con formación universitaria y alta cualificación, en particular con estudios centrados en género, violencia contra las mujeres, igualdad y feminismo.

La entrevista se estructuró en preguntas distribuidas en tres bloques temáticos que hacían referencia a la experiencia de militancia en el movimiento feminista, el uso y empleo de la tecnología y los repertorios de acción colectiva digitales empleados. La finalidad no era sólo realizar una aproximación desde un punto de vista temático sino identificar la generación de ideas más allá de procedimientos y técnicas analíticas específicas (Coffey; Atkinson, 2003, p. 19).

En la entrevista número 12 se alcanzó el punto de saturación del discurso en torno a las materias planteadas en el guion, por lo que se dio por finalizado el trabajo empírico ante la práctica iterativa de conceptos e ideas (Callejo, 1995, p. 96). Como indican Taylor y Bogdan (1987, p. 108), la saturación se distingue cuando al realizar entrevistas adicionales no se produce ninguna comprensión auténticamente nueva, limitándose la evolución de las propiedades de la categoría y adquiriéndose confianza empírica de que no van a surgir más discursos relevantes (Vallés citado por Glaser y Strauss, 1999, p. 215).

\section{Análisis}

\subsection{Escuelas de aprendizaje feminista, coaliciones puente y tecnología}

La Red no ha generado una activación de la militancia feminista. Un número destacado de las informantes seleccionadas tiene una trayectoria de participación política en el movimiento feminista amplio y continuado en el tiempo, destacando dos vías: 
- Una es la pertenencia (o al menos vinculación puntual) con partidos progresistas como el PSOE y de manera más reciente Podemos, así como con sus respectivos entornos (Federación de Mujeres Progresistas, Fundación Mujeres, Podemos feminismos...).

- Otra vía es la militancia en organizaciones de la sociedad civil y en el tejido asociativo centrado en exclusiva en el feminismo (Asamblea Feminista, Fórum de política feminista...).

Pese a las diferencias tácticas, estratégicas e ideológicas, así como a la progresiva erosión de los movimientos tradicionales de acción política feminista y la institucionalización de sus prácticas, no hay una renuncia a sus modos de hacer, sino una reivindicación de los mismos como "escuela de aprendizaje" y archivo de conocimientos. Este planteamiento ratifica la noción de Tarrow (2012, p. 235) sobre cómo los movimientos sociales describen un tipo de activismo puente lateral (con la creación de coaliciones formales entre organizaciones contemporáneas mediante los lazos que unen a activistas individuales) y en el tiempo (los puentes entre activistas sirven para que éstas se muevan de una organización a otra).

Pese a las diferencias tácticas, estratégicas e ideológicas, no hay una renuncia a sus modos de hacer, sino una reivindicación como "escuela de aprendizaje" y archivo de conocimientos

Sin embargo y como destaca Dalton (2007), el modo de actuar en política ya no consiste sólo en ir a una reunión o juntarse con amigos y vecinos para asistir a una marcha o a una manifestación; tampoco en la capacidad para crear coaliciones. Hoy en día desplegar habilidades informáticas es vital. El uso de la Red para la militancia feminista ha supuesto la creación de un espacio de encuentro relevante, fortaleciendo los puentes colectivos y sobre todo individuales, de las participantes en el movimiento.

En términos de acción, la Red ha suprimido algunas barreras creando coaliciones temporales a partir de la co-presencia digital. Respecto al conocimiento, la multiplicidad de fuentes informativas facilita un aumento de la reflexión en torno a cuestiones latentes en el movimiento. Ambas cosas contribuyen a que la eterna distinción entre los planos fuera y dentro de la Red parezca difuminada:

"Cada vez existe una mayor vinculación con otras activistas feministas. A través de las asambleas y reuniones que se convocan, hay gente con la que te reúnes y luego trabajas en las redes. Pero hay mucha gente que conoces de las redes y no personalmente" (Sujeto 1).

"No creo que haya una militancia distinta. Internet es una herramienta más. [...] Hacemos lo mismo pero más fluido, más ágil. Tenemos sentimiento de ser más" (Sujeto 4).

\subsection{Usos y espacios: Twitter vs. Facebook}

La noción de uso hace referencia a una acción estructurada y repetida en el tiempo, resultado de cierta historia colectiva, no individualizada, pero que a su vez otorga a los sujetos un marco de creatividad (Callejo, 1995). En el caso del ciberfeminismo en España se identifican cuatro usos predominantes que se corresponden con cuatro objetivos del movimiento en la Red:

- divulgación de convocatorias;

- organización de acciones;

- construcción de agenda;

- autoexpresión.

En el ciberfeminismo en España se identifican cuatro usos predominantes: divulgación de convocatorias, organización de acciones, construcción de agenda y autoexpresión

En todos los casos el espacio digital se concibe como un soporte y no como base de las acciones (Van-Laer; Van-Aelst, 2010), describiendo un modo de compromiso más institucional (Flanagin; Stohl; Bimber, 2006, pp. 34-36) y combinando la interacción directa y la no directa. Si bien en el caso de la divulgación de convocatorias, se sigue un patrón reconocido con pocos elementos de innovación, los otros tres usos tienen mayores cuotas de autonomía, auto-organización y horizontalidad gracias a la Red.

La realización de acciones puede evitar el filtro de organizaciones convencionales como partidos políticos $u$ asociaciones y amplificarse con redes digitales, al igual que las posibilidades de construir una agenda propia y luchar contra las exclusiones de las agendas política y mediática. Por último, cabría destacar las posibilidades autoexpresivas (Sánchez-Duarte; Magallón, 2016, p. 13) que concede la Red y su potencialidad para convertir opiniones individuales y cabreos personales volcados en la Red en estrategias efectivas de acción:

"Gracias a Twitter y a otras redes la frase 'yo sola no puedo, con amigas sí se hace más posible. Por ejemplo, si quiero boicotear a unas marcas que patrocinan el machismo, yo sola no puedo interpelarlas. En internet creas redes activas. Si todas sabemos que una de nosotras lo va a hacer y le decimos a las marcas y a los medios de comunicación: 'os estamos vigilando, no voy a consumir esta marca', será efectivo” (Sujeto 6).

Uno de los debates más relevantes y que presentaba visiones contrapuestas entre nuestras informantes hace referencia a qué espacios digitales emplear para la acción política en Red. Si para un número significativo de las entrevistadas Twitter es el espacio más adecuado para la influencia política, para otras Facebook genera una comunidad más cohesionada y el ejercicio de un debate feminista más relevante: 
"Cada una tiene que saber cuál es su herramienta. Twitter es la que en estos momentos marca posición política y es abierto. Facebook es endogámico. Estamos hablando entre nosotras en Facebook. Lo que tenemos que hacer es intervenir en Twitter, saber participar en las conversaciones en Twitter, pelear por nuestras posiciones en Twitter, porque son las posiciones que se reflejan en los medios de comunicación tradicionales" (Sujeto, 2).

"Tenemos más impacto en FB porque tenemos más idea de comunidad que de revista" (Sujeto 3).

\section{Las entrevistadas no practican un acti- vismo diferente en la Red, sino que en- tienden que espacios como Facebook o Twitter han amplificado y facilitado la capacidad de movilización colectiva}

Por último, habría que destacar el planteamiento expuesto respecto al encapsulamiento del activismo feminista en redes como Twitter y Facebook. Sin restarle importancia a su potencialidad, se contempla la necesidad de superar (o al menos complementar) los actos más superficiales y con poca incidencia política, como por ejemplo retweetear, por otros con mayor contenido y reflexión. Así se plantea la creación de espacios propios, webs, repositorios, medios plenamente feministas o incluso la realización de entradas en Wikipedia. Frente a las limitaciones de tiempo y la caducidad de las acciones digitales, se dispondría de espacios más permanentes para evitar el ruido discursivo de las redes, asentar contenidos y generar un conocimiento compartido más reposado rompiendo la brecha de posicionamiento y habilidad (Del-Fresno, 2016) y formando "espacios refugio" feministas en red:

"En el 2009 comprobamos también que para aquellos temas especiales eran necesarios nuestros espacios propios, nuestro repositorio de imágenes, nuestro blog, nuestra web [...] Nos han permitido ser visibles y hacer escuchar nuestras voces, pero además es un gran espacio de construcción de conocimiento colectivo. Uno de los déficits que tenemos desde el activismo feminista es dedicar poco tiempo a esto. Dedicamos mucho al "chucuchucu" de las redes y poco a asentar contenidos" (Sujeto 2).

\subsection{Debilidades}

Los usos y tácticas del ciberactivismo feminista tienen algunas debilidades ampliamente recogidas en las opiniones de las informantes seleccionadas. Si bien no se puede hablar en general de pesimismo, se aprecia una aproximación crítica a los modos de hacer acción política feminista en la Red.

En primer lugar, la endogamia discursiva de las prácticas digitales puede favorecer una continua retroalimentación en la que un amplio número de participantes no implique una pluralidad de voces y materias. Como indicaba una de las entrevistadas:

“A mí me preocupa que caigamos en el hedonismo, que estemos más preocupadas hablando de poliamor que de la Ley de dependencia" (Sujeto 3).

Esta expresión conecta con la construcción de enclaves deliberativos digitales expuesto por Sunstein (2010), que si bien fortalecen una identidad cohesionada, podrían incapacitar el intercambio discursivo con otras comunidades y polarizar los argumentos (Mancini, 2013).

De igual modo, la obsesión por estar en todos los espacios digitales y participar en la gran mayoría de debates restaría fortalezas, fragmentando en exceso la acción. En este contexto y valorando la lógica de visibilidad en red se podrían generar líderes del movimiento construidos a partir de redes como Twitter. Como indican Sousa, Pinto y Costa-e-Silva $(2013$, p. 11), la lucha por la atención tal vez esté derivando en repetir la pauta por la que los más vistos acaban siendo los más visitados, impidiendo una verdadera esfera pública plural.

\section{Es un tipo de subactivismo que se arrai- ga en las rutinas de las activistas y en la organización de su vida social cotidiana}

Para algunas entrevistadas la solución a este tipo de debilidades pasa por concebir la Red como un espacio de disputa y no como un territorio propio. Frente a la reacción debería ser potenciada la proposición, reapropiándose del discurso feminista ("-Ahora todo el mundo es feminista, está de moda y eso es contraproducente" (Sujeto 7)), normalizado a través de la institucionalización de las voces y las prácticas digitales y saliendo de espacios puramente comerciales (como por ejemplo Facebook):

"También descubrimos la otra cara de la moneda. La agenda feminista. Asumir que en las redes sociales somos invitadas, no es nuestro territorio, y son muy vulnerables a ciertas posiciones. Pero igual nosotras podemos empezar a hacer clics de peligrosidad a todo el sector que está amenazando desde el machismo radical, desde las situaciones que generan violencia de género. A veces mantenemos una posición pasiva pero no somos conscientes de las posibilidades que las redes sociales son un espacio en disputa. No siempre tenemos la posición activa de disputarlo. Es importante no ser activas si no reactivas" (Sujeto 2 ).

\section{Conclusiones}

Frente al determinismo tecnológico, y como señala Sánchez-Duarte (2016, p. 42), la importancia de internet no reside en establecer un vínculo directo entre conexión y calidad/cantidad de la participación, sino en articular un espacio favorable para constituir actos políticos. En el ciberactivismo feminista en España se identifica un espacio en el que se encuentran militancias previas y repertorios reconocidos digitalizados. Este hecho conforma un patrón conocido de fácil activación en red ante determinadas acciones y sin la necesidad de unidades centrales, organizaciones convencionales o núcleos de participación. Este es un tipo de subactivismo que opera en la experiencia subjetiva de las activistas 
feministas y que se arraiga en sus rutinas y en la organización de su vida social cotidiana.

La Red ha generado por tanto un continuum entre la militancia feminista convencional y la online. Las entrevistadas no asumen practicar un activismo diferente en la Red, sino que entienden que espacios como Facebook o Twitter han amplificado y facilitado la capacidad de movilización colectiva. Esto se traduce en la creación de fuertes coaliciones para realizar acciones de presión muy efectivas gracias a su difusión en redes².

\section{Si bien la Red potencia la sensación de comunidad, democratiza la divulgación y da visibilidad, una de las autocríticas es la necesidad de apropiarse del discurso feminista y crear contenido}

La Red se utiliza prioritariamente como soporte para dar a conocer acciones concretas y divulgar convocatorias, más que para construir asuntos o debatirlos. Si bien potencia la sensación de comunidad y la democratización de la divulgación y visibilidad de sujetos feministas más diversos, una de las autocríticas es la necesidad de reapropiarse del discurso feminista en redes sociales y crear contenido compartido. No se trata por tanto de estar presentes en las redes solamente para hacer circular discursos fragmentados que pudieran ser endogámicos y autorreferenciales, sino de evitar el fetichismo tecnológico (Dean, 2005), generando espacios propios y formulando propuestas y discursos reactivos que adquieran carácter político.

Como proponen algunas activistas, esto supone concebir la Red como un espacio de disputa y no como un territorio propio. De este modo los medios sociales y en especial Twitter serían escenarios y herramientas para una política latente feminista a partir de la cual emprender acciones fuera, pero sobre todo dentro de la Red; así como activar modos de compromiso emprendedores y patrones de interacción personales y directos. En suma, se trataría de ejercer una actividad política modulable y adaptativa que contribuya a fomentar el compromiso social y cívico con las reivindicaciones feministas.

\section{Notas}

1. Este artículo forma parte de los proyectos financiados por el Ministerio de Economía y Competitividad español CSO2013-43960-R y CSO2016-77331-C2-1-R

2. Como hitos se referenciaban las acciones del "Tren de la libertad" (contra la reforma de la Ley del aborto en 2014) o el Paro internacional de mujeres (8 de marzo de 2017).

\section{Bibliografía}

Amnå, Erik; Ekman, Joakim (2014). "Standby citizens: diverse faces of political passivity". European political science review, v. 6, n. 2, pp. 261-281.

http://dx.doi.org/10.1017/S175577391300009X

Bakardjieva, Maria (2009). "Subactivism: Lifeworld and politics in the age of the internet". The information society, v.
25, n. 2, pp. 91-104.

http://dx.doi.org/10.1080/01972240802701627

Baym, Geoffrey; Shah, Chirag (2011). "Circulating struggle". Information, communication \& society, v.14, n. 7, pp. 10171038.

http://dx.doi.org/10.1080/1369118X.2011.554573

Beck, Ulrich (2002). La sociedad del riesgo global. Madrid: Siglo XXI. ISBN: 9788432312618

Benkler, Yochai (2014). La riqueza de las redes. Cómo la producción social transforma los mercados y la libertad. BarceIona: Icaria. ISBN: 9788498886344

Bennett, Lance (1998). "The uncivic culture: Communication, identity, and the rise of lifestyle politics". Political science \& politics, v. 31, n. 4, pp. 741-761. https://doi.org/10.1017/S1049096500053270

Bennett, Lance; lyengar, Shanto (2010). "A new era of minimal effects? The changing foundations of political communication". Journal of communication, v. 58, n. 4, pp. 707-731. http://dx.doi.org/10.1111/j.1460-2466.2008.00410.x

Bimber, Bruce (2003). Information and American democracy: Technology in the evolution of political power. Cambridge: Cambridge University Press. ISBN: 9780521800679

Callejo, Javier (1995). "Elementos para una teoría sociológica del consumo". Papers: Revista de sociología, v. 47, pp. 75-96.

Chadwick, Andrew (2007). "Digital network repertoires and organizational hybridity". Political communication, v. 24, n. 3, pp. 283-301.

http://dx.doi.org/10.1080/10584600701471666

Coffey, Amanda; Atkinson, Paul (2003) Encontrar el sentido a los datos cualitativos. Estrategias complementarias de investigación. Medellín: Ed. Contus. ISBN: 958 655631x

Coleman, James (1988). "Social capital in the creation of human capital". The American journal of sociology, v. 94, pp. 95-120.

Crouch, Colin (2005). Post-democracy. Cambridge: Polity Press. ISBN: 9780745633145

Dahlberg, Lincoln (2007). "Rethinking the fragmentation of the cyberpublic: from consensus to contestation". New media society, v. 9, n. 5, pp. 827-847. https://doi.org/10.1177/1461444807081228

Dalton, Russel (2007). Citizen politics: Public opinion and political parties in advances industrial democracies. Washington DC: CQ Press. ISBN: 9781452203003

Dean, Jodi (2005). "Communicative capitalism and the foreclosure of politics". Cultural politics, v. 1, n. 1, pp. 51-74 http://dx.doi.org/10.1080/08935696.2015.1007792

Del-Fresno, Miguel (2016). Netnografía. Investigación, análisis e intervención social. Barcelona: UOC. ISBN: 97884 97883856

Ekman, Joakim; Amnå, Erik (2012). "Political participation and civic engagement: Towards a new typology". Human affairs, v. 22, n. 3, pp. 283-300. 
https://doi.org/10.2478/s13374-012-0024-1

Flanagin, Andrew; Stohl, Cynthia; Bimber, Bruce (2006). Modeling the structure of collective action. Communication monographs, v. 7, n. 1, pp. 29-54.

http://dx.doi.org/10.1080/03637750600557099

Giddens, Anthony (1991). Modernity and self-identity. Self and society in the late modern age. Stanford: Stanford University Press. ISBN: 9780804719445

Heaney, Michael; Rojas, Fabio (2014). “Hybrid activism: Social movement mobilization in a multimovement environment". American journal of sociology, v. 119, n. 4, pp. 1047-1103.

https://doi.org/10.1086/674897

Holzer, Boris; Sørensen, Mads (2003). "Rethinking subpolitics: Beyond the 'iron cage' of modern politics?". Theory culture \& society, v. 20, n. 2, pp. 79-102.

http://dx.doi.org/10.1177/0263276403020002005

Johnson, Thomas; Bichard, Shannon; Zhang, Weiwu (2009). "Communication communities or "cyberghettos?": A path analysis model examining factors that explain selective exposure to blogs". Journal of computer-mediated communication, v. 15 , n. 1 , pp. 60-82.

https://doi.org/10.1111/j.1083-6101.2009.01492.x

Lim, Merlyna; Kann, Mark (2008). "Politics: Deliberation, mobilization, and networked practices of agitation". In: Varnelis, Kazys (ed.). Networked publics, pp. 78-107. ISBN: 978 0262517928

Mancini, Paolo (2013). “Media fragmentation, party system and democracy". The international journal of press/politics, v.18, n.1, pp. 44-60.

http://dx.doi.org/10.1177/1940161212458200

McCarthy, John (1999). "Adoptar, adaptar e inventar límites y oportunidades". En: McAdam, Dough; McCarthy, John; Zald, Mayer. Movimientos sociales: perspectivas comparadas, pp. 205-220. ISBN: 8470903314

Mozorov, Evgeny (2011). The netdesilusion. The dark side of Internet freedom. New York: Public Affairs. ISBN: 1610391063.

Neuman, Russell; Bimber, Bruce; Hindman, Matthew (2010). "Internet and four dimensions of citizenship". In: Shapiro, Robert; Jacobs, Lawrence. The Oxford handbook of american public opinion and the media, pp. 22-43. ISBN: 978 0199545636

Neumayer, Christina; Svensson, Jakob (2016). "Activism and radical politics in the digital age: Towards a typology". Convergence, v. 22, n. 2, pp. 131-146.

http://dx.doi.org/10.1177/1354856514553395

Núñez-Puente, Sonia; Fernández-Romero, Diana; Peña, Palma (2016). "Ciberactivismo contra la violencia de género: fetichismo tecnológico e interactividad". Feminismo/s, v. 27, pp. 177-195.

http://dx.doi.org/10.14198/fem.2016.27.10

Núñez-Puente, Sonia; Fernández-Romero, Diana; Vázquez-Cupeiro, Susana (2015). "El impacto de Internet como herramienta de lucha contra la violencia de género. Análisis de la brecha digital y de los procesos de victimización". Revista europea de derechos fundamentales, v. 26, pp. 65-77. http://journals.sfu.ca/redf/index.php/redf/article/view/194

Núñez-Puente, Sonia; Vázquez-Cupeiro, Susana; Fernández-Romero, Diana (2016). "Ciberfeminismo contra la violencia de género: análisis del activismo online-offline y de la representación discursiva de la víctima". Estudios sobre el mensaje periodístico, v. 22, n. 2, pp. 861-877. http://dx.doi.org/10.5209/ESMP.54240

Papacharissi, Zizi (2009). "The virtual sphere 2.0: The internet, the public sphere and beyond". In: Andrew Chadwick, Philip Howard. Routledge handbook of internet politics, pp. 230-245. ISBN: 9780415429146

Plant, Sadie (1997). Zeros and ones: Digital women and the new technoculture. London: Fourth Estate. ISBN: 1857026985

Putnam, Robert (1995). “Bowling alone: America's declining social capital". Journal of democracy, v. 6, n. 1, pp. 65-78.

Robertson, Scott; Vatrapu, Ravi; Medina, Richard (2010). "Off the wall political discourse: Facebook use in the 2008 U.S. presidential election". Information polity, v. 15, n. 1, pp. 11-31.

http://dx.doi.org/10.3233/IP-2010-0196

Sádaba, Igor (2012). “Acción colectiva y movimientos sociales en las redes digitales". Arbor. Ciencia, pensamiento y cultura, v. 188, pp. 781-794.

http://dx.doi.org/10.3989/arbor.2012.756n4011

Sánchez-Duarte, José-Manuel (2016). “The Net as a space for political militancy: technology and participation in the electoral campaign". Communication \& society, v. 29, n. 3, pp. 33-47.

http://dx.doi.org/10.15581/003.29.3.sp.33-47

Sánchez-Duarte, José-Manuel; Magallón, Raúl (2016). “Estrategias de organización y acción política digital". Revista de la asociación española de investigación de la comunicación, v. 3, n. 5, pp. 9-16.

http://www.revistaeic.eu/index.php/raeic/article/view/115/96

Scott, Alan; Street, John (2000). "From media politics to e-protest. The use of popular culture and new media in parties and social movements". Information, communication \& society, v. 3, n. 2, pp. 215-240.

https://doi.org/10.1080/13691180050123712

Sousa, Helena; Pinto, Manuel; Costa-e-Silva, Elsa (2013). "Digital public sphere: Weaknesses and challenges". Comunicação e sociedade, v. 23, pp. 9-12.

http://dx.doi.org/10.17231/comsoc.23(2013).1630

Sunstein, Cass (2007). Republic.com. 2.0. Princeton, NJ: Princeton University Press. ISBN: 9780691143286

Tarrow, Sidney (2012). El poder en movimiento. Los movimientos sociales, la acción colectiva y la política. Madrid: Alianza Editorial. ISBN: 9788420641911

Taylor, Steve; Bogdan, Robert (1987). Introducción a los métodos cualitativos de investigación. La búsqueda de signifi- 
cados. Buenos Aires: Paidós. ISBN: 8475098169

Tilly, Charles (2009). Los movimientos sociales. 1768-1999. Desde sus orígenes a Facebook. Madrid: Libros de Historia. ISBN: 9788498920451

Treré, Emiliano; Cargnelutti, Daniele (2014). "Movimientos sociales, redes sociales y web 2.0: el caso del Movimiento por la Paz con Justicia y Dignidad". Communication \& society, v. 27, n. 1, pp. 183-203

http://www.unav.es/fcom/communication-society/es/ articulo.php?art_id $=486$

Vallés, Miguel (1999). Técnicas cualitativas de investigación social. Reflexión metodológica y práctica profesional. Madrid: Síntesis. ISBN: 8477384495
Van-Aelst, Peter; Walgrave, Stefaan (2002). "New media, new movements? The role of the internet in shaping the "Anti-globalization" movement". Information, communication and society, v. 5, n. 4, pp. 465-493. http://dx.doi.org/10.1080/13691180208538801

Van-Laer, Jeroen; Van-Aelst, Peter (2010). "Internet and social movement action repertoires". Information, communication \& society, v.13, n. 8, pp. 1146-1171.

http://dx.doi.org/10.1080/13691181003628307

Wajcman, Judy (2000). "Reflections on gender and technology studies: In what state is the art?". Social studies of science, v. 30, n. 3, pp. 447-464.

http://dx.doi.org/10.1177/030631200030003005

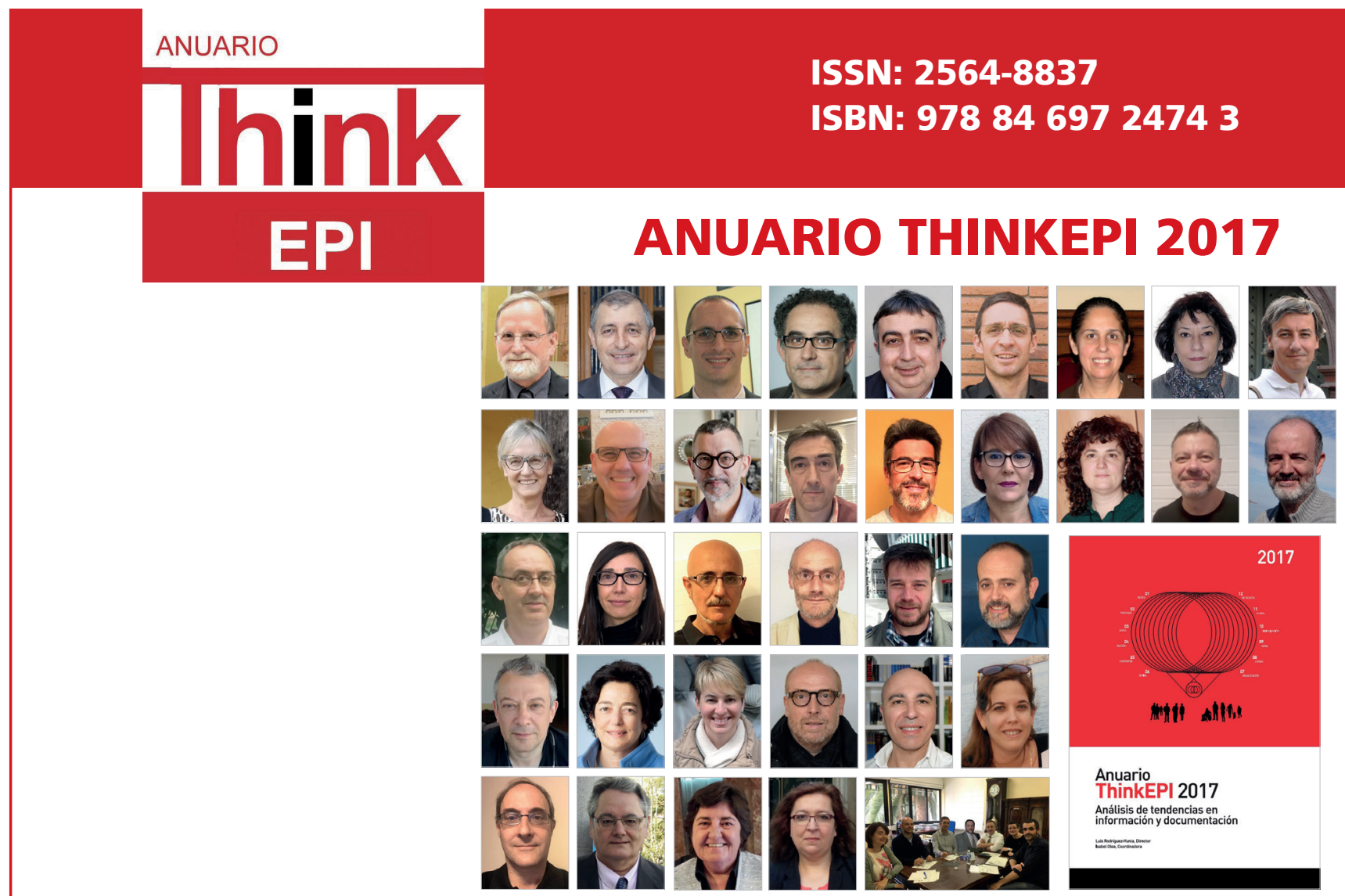

\section{PRECIOS ANUARIO THINKEPI}

Suscripción online (2007-2017)

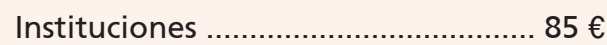

Números sueltos

Instituciones

Anuario ThinkEPI 2017 (pdf)

Anuario de ańos anteriores $20 €$

Individuos (particulares)

Anuario ThinkEPI 2017 (pdf)

Anuario de ańos anteriores
Es posible el acceso mediante suscripción a todos los Anuarios ThinkEPI publicados hasta el momento desde el Recyt de la Fecyt http://recyt.fecyt.es/index.php/ThinkEPI

\section{Más información: Isabel Olea epi.iolea@gmail.com}

